\title{
Über Kosten sprechen und Verantwortung für den kranken Menschen übernehmen
}

\author{
Der beste Spareffekt im Gesundheitswesen kann nur von den Ärzten selbst erreicht \\ werden. Sie wissen um die gesundheitlichen Probleme ihrer Patienten und kennen \\ die Angebote der modernen Medizin, diese zu lösen. Sie wissen aber auch diese \\ Angebote zu relativieren. Kosten sparen nur anderen zu überlassen, ist verantwor- \\ tungslos.
}

Hanswerner Iff

Ehemals Chefarzt der Solothurnischen Höhenklinik Allerheiligenberg

Korrespondenz:

Dr. med. Hanswerner Iff Berchtoldstrasse 43 CH-3012 Bern

hw@iffs.org
Unsere politischen Instanzen haben die Einführung der Fallpauschalen (Swiss DRG) in den Spitälern ab 2012 beschlossen und sind nun dabei, integrierte Ärztenetzwerke (ManagedCare) gesetzlich zu verankern. Bei beiden Modellen spielen sowohl medizinische als auch ökonomische Abwägungen eine Rolle, wobei Ärztinnen und Ärzten eine entscheidende Rolle zukommt. DRG und Managed Care werden als «Gesundheitskosten-Zunahme-Bremse» gelobt, obschon einerseits die ökonomische Wirkung nicht vorhersagbar ist und andererseits der gerechte Zugang zu medizinischen Gütern auf dem Spiel steht. Es soll so sein, dass wir Ärztinnen und Ärzte auch bei der ökonomischen Seite einer vernünftigen Entwicklung unseres Gesundheitswesens tatkräftig mithelfen. Dies ist richtig. Dafür müssen wir aber mehr über Kosten sprechen. Es drängt, dass wir dies auch als Ärzte tun.

Während meiner Arbeitszeit pflegte ich, spezielle Untersuchungen - zu deren Veranlassung ich meinen ärztlichen Mitarbeitern die Freiheit gab -, die aber in der Folge nicht weiter halfen, mit «selbst bezahlen, bitte» zu kommentieren. Diese Worte gaben immer wieder Gelegenheit, über Kosten, die in der täglichen Arbeit und am Krankenbett verursacht werden, zu diskutieren. Als Erinnerung an diese Zeit blieb mir, als Geschenk einiger Assistenzärztinnen und -ärzte, ein Stempel mit rotem Stempelkissen mit den Worten «Sälber zahle». Vor nicht zu langer Zeit führte ich ein Gespräch mit einem Assistenzarzt in klinischer Ausbildung, der mir meine Frage, ob in seinem beruflichen Alltag auch über Kosten, die durch ihn am Krankenbett verursacht werden, gesprochen werde, mit einem dezidierten «nie» beantwortete. Vor zwei Jahren fragte ich, selbst Patient in einem Universitätsspital, einen Assistenzarzt, ob er wisse, was eine Bluttransfusion kosten würde. Der junge Kollege wollte mir, bei klinisch gutem Befinden, aber einem Hb-Wert unter einer spitalintern festgelegten Indikationsgrenze für eine Bluttransfusion, ein Erythrozytenkonzentrat geben. Grosses Staunen über diese Frage, doch keine Ahnung über die Kosten seines Tuns! Ich bin der Meinung, dass sich das ändern muss. Kostenüberlegun- gen gehören in den medizinischen Alltag. So freut es mich, dass die diesjährige Jahrestagung der SGIM unter dem Leitthema «Komplexität, Konsum, Kosten» stattfand. Denn um alle Ideen, wie eine vernünftige Kostendiskussion anzukurbeln wäre, sollten wir dankbar sein.

Vor Jahren, 1981, habe ich unter dem Titel «Teure Medizin» in einer Kolumne [1] zu den bereits damals politisch geforderten Sparaufträgen an die medizinische Versorgung, folgendes geschrieben: «Ich bin der Meinung, dass der beste Spareffekt im Gesundheitswesen nur von den Ärzten selbst erreicht werden kann. Der Arzt weiss um die gesundheitlichen Probleme seiner Patienten und kennt die Angebote, die die moderne Medizin macht, diese zu lösen. Insbesondere weiss er auch, diese Angebote zu relativieren: «was muss ich einsetzen, was könnte ich einsetzen

\section{Parler des coûts et assumer ses respon-} sabilités à l'égard des personnes malades Une partie non négligeable des coûts de la santé apparaît lors de la prise en charge par le médecin. Pour mieux comprendre ces coûts et pouvoir en débattre, le médecin doit avoir connaissance de la valeur et du prix de ses prestations. Ce savoir doit être acquis par une formation pendant les études et la formation continue. Renoncer à certains coûts requiert un certain savoir, et donc une formation adéquate, mais également un sens prononcé des responsabilités. Refuser un acte médical parce qu'il est trop coûteux serait contraire à l'éthique médical.

Aux Etats-Unis, depuis longtemps nos maîtres à penser en médecine, le désir de mieux contrôler les coûts en dehors des DRG et du managed care semble vouloir s'affirmer. Mais ce contrôle ne peut être uniquement confié aux caisses-maladies ou à l'Etat (Confédération et cantons) ou aux organisations de patients. Ceci mettrait en danger le patient et irait à l'encontre du sens des responsabilités du médecin. 
(tue es aber aus diesen medizinischen und jenen finanziellen Gründen nicht), was ist sicher nicht nötig einzusetzen>. So hat der Arzt x-mal an einem einzigen Tag Gelegenheit zu sparen und trotzdem eine gute Medizin zu betreiben.» Diese Aussage wurde nicht nur wohlwollend aufgenommen. Der Arzt, die Ärztin als Verdiener und Sparer bleibt ein Paradox. Preise festzulegen für therapeutisches Handeln ist äusserst schwierig, weil sowohl kostspielige Verfahren wie weiser Verzicht auf Massnahmen sowohl Erfolg wie Misslingen bringen können.

Interessanterweise kommen, auch im Zusammenhang mit der Gesundheitsreform in den USA, Vorschläge, die sich eingehend mit der Kostenfrage im medizinischen Alltag abgeben und meine, vor fast dreissig Jahren geäusserte Idee unterstützen.

\section{Patienten in Gegenden mit tiefen Gesundheitskosten sind weder gesünder noch kränker als in solchen mit hohen Kosten}

Unter dem Titel «Doctors as the Key to Health Care Reform» forderte A. S. Relman kürzlich im New England Journal of Medicine (NEJM) [2] Ähnliches. Der Autor ortet das Hauptproblem für den übermässigen Kostenzuwachs im amerikanischen Gesundheitswesen dort, wo es teilweise auch in der Schweiz liegt. Ich zitiere aus dem Original: «Most doctors are paid on a fee-for-service basis, which is a strong incentive for them to maximize the elective services they provide. This incentive, combined with the continued introduction of new and more expensive technology, is a major factor in driving up medical expenditures. The same incentive is attracting more and more young doctors into specialities that command much higher fees - and therefore guarantee much greater income than those earned by primary care practitioners. Primary care is rapidly becoming an endangered speciality; an important, but not the only, reason is its relatively low economic rewards.»
Im weiteren macht der Autor in klaren Worten darauf aufmerksam, dass der Gesundheitsmarkt kein üblicher Markt ist und Wettbewerb nicht zu tieferen Kosten führt. Er bedauert, dass dieser Umstand von den politischen Instanzen nicht erkannt wird. Ärztinnen und Ärzte im Kontakt mit ihren Patienten würden die Kosten bestimmen, nicht Versicherungen, Gesetzgeber und Gesetze oder Staatsbeamte. Ähnliche Überlegungen macht sich $\mathrm{H}$. Brody in einem weiteren Artikel im NEJM [3]. Er sieht die Ärzte nicht als unschuldige Zuschauer (innocent bystanders) in der Kostenentwicklung im Gesundheitswesen und schlägt vor, dass jede Fachorganisation fünf kostspielige Dienstleistungen, die dem Patienten nach der wissenschaftlichen Evidenzlage keinen wesentlichen Benefit bringen würden, eliminieren sollte. Der Autor sieht eine ethische Verpflichtung des Ärztestandes welche Krankenversicherungen, Gesetzgeber oder Beamten nicht erbringen können - die Interessen des Patienten durch tiefe Kosten zu wahren. Auch macht er auf einen Umstand aufmerksam, der auch in der Schweiz zu mehr Diskussionen Anlass geben sollte: Patienten in «lower-cost regions», also Gegenden der USA mit tiefen Gesundheitskosten, seien weder gesünder noch kränker als solche in «higher-cost regions». In diesem Umstand müsse Sparpotential liegen. Dieses läge ganz in den Händen der Ärzte. Die Tatsache, dass z. B. der Bewohner des Chablais vaudois weniger Krankenkassenprämien bezahlt als derjenige des Chablais valaisan, weist darauf hin, dass solche Kostenunterschiede auch in der Schweiz vorhanden sind.

A. S. Relman plädiert zudem in seinem Artikel für eine Kosteneindämmung unter dem Kürzel ACO, ausgeschrieben Accountable $\underline{\text { Care }}$ Organisation. Es mag weise sein, das Kürzel von Anfang an zu definieren, um Unklarheiten, die sich mit Fremdwörtern ergeben, zu vermeiden. "Accountability» wird im Mosby's Medical Dictionary (2009) mit «Responsibility for the moral and legal requirements of proper patient care» definiert, von den möglichen Übersetzungen von «accountable» erscheint mir diejenige mit «verantwortlich» oder «rechenschaftspflichtig» am besten. Bemerkenswert ist, dass in beiden Artikeln weder von Health Maintenance Organisation (also HMO), noch von

\section{Die Kostenkontrolle nur den Krankenkassen, dem Staat, den Kantonen oder den Patientenorganisationen zu übergeben, gefährdet den kranken Menschen und ist verantwortungslos}

Und weiter: «A system like ours, which is grossly deficient in primary care physicians and dominated by specialists who are trained to use expensive tests and procedures, is inevitably costly, particularly when most specialists practice as independent small business, competing for patient referrals and for income.»
Fallpauschalen (also DRG) oder von Managed Care gesprochen wird. Bekannt ist aber, dass diese in den USA zum teuersten Gesundheitswesen - und dabei noch zum unsozialsten - geführt haben.

Auch ich bin der Meinung, dass die medizinische Versorgung in erster Linie gegenüber dem Patienten 
und der Patientin, dann gegenüber der Gesellschaft und der medizinischen Lehre, verantwortbar sein soll. Für den Kranken soll sie wirksam sein, für die Gesellschaft preiswert und in der Lehre rational. Einzuräumen ist, dass das wissenschaftlich begründete und für den Kranken das Beste nicht immer bezahlbar ist. Darüber sollen aber nicht Krankenkassen mit einer Budgetlimite entscheiden, sondern ärztliche Gespräche, die zwar schwierig, aber oft nicht zu umgehen sind. Das gesundheitsrelevante und effiziente medizinische Vorgehen, dies sowohl im Spital wie in der ambulanten Medizin, bei Erkrankungen und in der Gesundheitsberatung, muss ein Problem bleiben, das sich an einzelnen Menschen orientiert. Die Ärztin und der Arzt und, wo immer sie auch arbeiten, müssen sich bewusst sein, dass eine Kostenkontrolle zu ihren Aufgaben gehört. Wenn die Sorge um die Kosten nur den Krankenkassen, den Gesundheitsökonomen, den Patientenorganisationen, den Spital- und den Sanitätsdirektoren überlassen wird, geht der wichtigste Aspekt unserer Arbeit, eben derjenige, bei dem es ums Kranksein und den kranken Menschen geht, verloren.

Es ist bemerkenswert, dass aktuell in der Schweiz ohne gesetzliche Grundlagen immer mehr vernetzte medizinische Institutionen entstehen [4]. Ärztinnen und Ärzte suchen nach gangbaren Lösungen, dort wo Schwierigkeiten bestehen, eine regionale medizinische Versorgung bereitzustellen (siehe z.B. den Leserbrief von U. Castelberg in [5]). Der gesellschaftspolitische Wandel, der vom Arzt und namentlich von der Ärztin keine Arbeitsexzesse - aber auch keine Einkommensexzesse - und damit geregelte Arbeit fordert und somit mehr Frauen den Arztberuf ermöglicht, begünstigt diese Arbeitsteilung. Diese wird durch neue Informationstechnologien unterstützt und führt gleichzeitig zu mehr Wissen und Erfahrung und ist Garant für

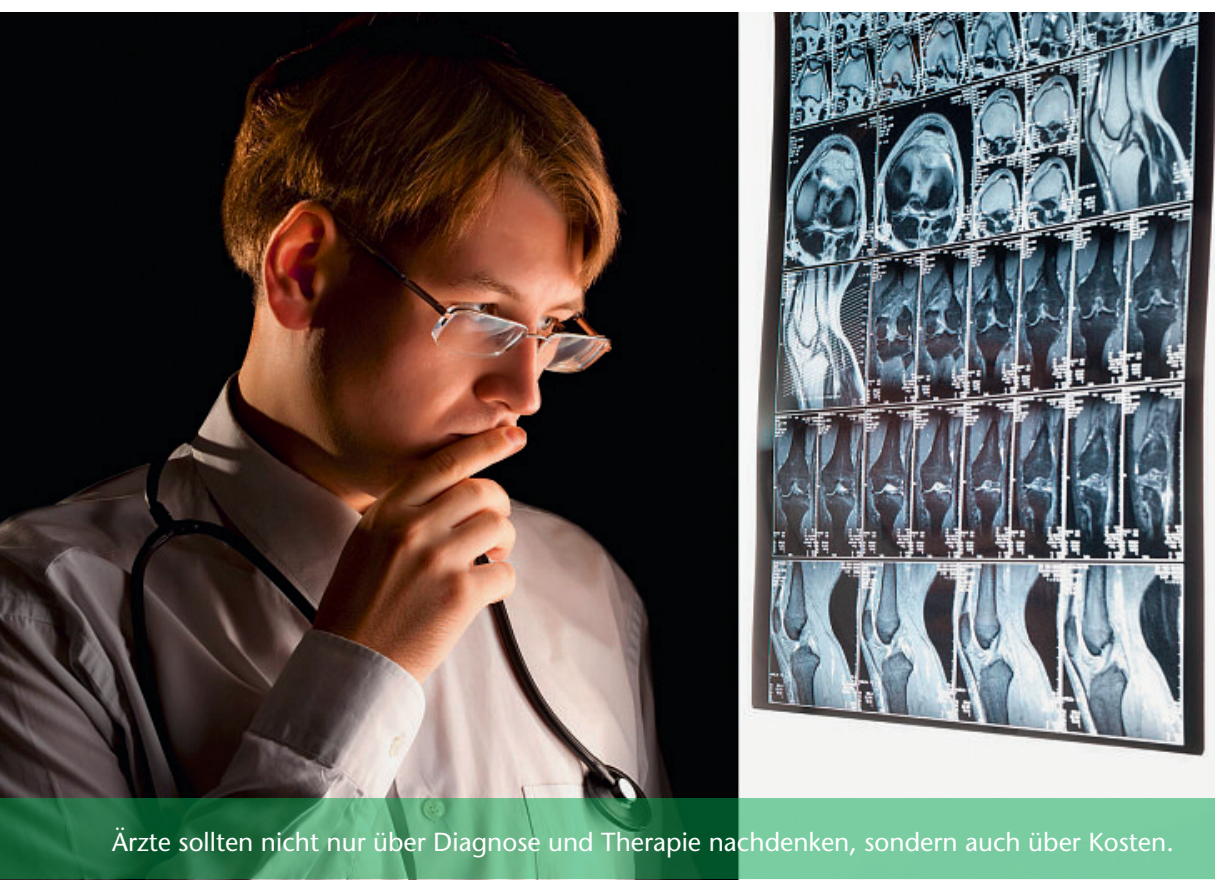

gleichbleibende gute Qualität. Damit beantwortet sich die Frage, ob wir wirklich die Krankenkassen als Organisatoren und Kontrolleure der Arztpraxen brauchen.

Es muss aber sichergestellt sein, dass Kostenüberlegungen bereits in der Grundausbildung der angehenden Mediziner und Medizinerinnen geübt und fester Bestandteil in der Weiterbildung werden. Diese sollen nicht nur auf Kosten-Nutzen-Studien basieren, sondern sich auch mit dem Teil des ärztlichen Gesprächs befassen, das bei einer medizinischen Kostenkontrolle geführt werden muss. Eine Verweigerung einer medizinischen Handlung, ein «Nein» also, braucht ein grosses Können, Verantwortung und auch Erfahrung. Dieses Können und das Übernehmen von Verantwortung müssen geschult, die Erfahrung muss weitergegeben werden. Basiert ein ablehnender Entscheid nur auf dem Hinweis, dass kein Geld mehr zur Verfügung steht, ist dies mit medizinischer Ethik, aber auch mit guter Gesundheitspolitik nicht vereinbar.

\section{Zusammenfassung}

- Der wesentlichste Teil der Gesundheitskosten entsteht im ärztlichen Kontakt mit Patienten und Patientinnen.

- Um diese Kosten besser verstehen, diskutieren und ein Gespräch darüber zu führen zu können, braucht es insbesondere bei Ärztinnen und Ärzten mehr Kenntnisse und demzufolge Ausbildung in Studium und Weiterbildung.

- Verzicht auf Kosten braucht nicht nur Ausbildung, sondern auch grosses Verantwortungsbewusstsein.

- Die Verweigerung einer medizinischen Handlung nur mit dem Hinweis auf das fehlende Geld ist mit der ärztlichen Ethik nicht zu vereinbaren.

- Aus den USA, in den letzten Jahrzehnten zweifellos unser Vorbild für medizinische Neuerungen, kommen Bestrebungen für eine Kostenkontrolle ausserhalb von DRG und Managed Care.

- Die Kostenkontrolle nur den Krankenkassen oder dem Staat und den Kantonen oder den Patientenorganisationen zu übergeben, gefährdet den kranken Menschen und ist verantwortungslos.

\section{Literatur}

1 Iff HW. Oltner Tagblatt. Meine Meinung über «Teure Medizin». Februar;1981.

2 Relman AS. Doctors as the Key to Health Care Reform. New Engl J Med. 2009;361:1225-7.

3 Brody H. Medicine's Ethical Responsability for Health Care Reform - The Top Five List. New Engl J Med. 2010;362:283-5.

4 Berchtold P, Peier K, Peier C. Erfolgreiche Entwicklung der Ärztenetzwerke in der Schweiz. Schweiz Ärztezeitung. 2008;89(47):2038-41.

5 Castelberg U. Drohender Versorgungsnotstand: agieren statt reagieren. Schweiz Ärztezeitung. 2009;90:(48):1879-80. 